\title{
Giant adenocarcinoma of unknown origin penetrating from the pelvic cavity to subcutaneous tissue: A case report and review of the literature
}

\author{
YA-FEI ZHANG ${ }^{1,2}$, ZHI-GUO RAO $^{1}$, JIAN-FEI GAO ${ }^{1}$, PING-GUANG XIA ${ }^{3}$, \\ DIAN-CHUN FANG ${ }^{2}$ and BI-CHENG ZHANG ${ }^{1}$
}

\begin{abstract}
Departments of ${ }^{1}$ Oncology and ${ }^{3}$ Orthopaedic Surgery, Wuhan General Hospital of Guangzhou Command, People's Liberation Army, Wuhan 430070; ${ }^{2}$ Department of Gastroenterology, Southwest Hospital, Third Military Medical University, Chongqing 400038, P.R. China
\end{abstract}

Received February 6, 2012; Accepted April 24, 2012

DOI: 10.3892/ol.2012.705

\begin{abstract}
In this study, we present a case of a 43-year-old female patient who was admitted to our hospital due to a giant mass on the left buttock. Imaging tests revealed that the mass was a solid-cystic tumor with a large size of 143x430x180 mm, penetrating from the pelvic cavity to the subcutaneous tissue. Pathology tests indicated a metastatic mucinous adenocarcinoma which was most likely of gastrointestinal origin. However, there was no evidence to confirm the existence of malignant changes in the gastrointestinal tract.
\end{abstract}

\section{Introduction}

In clinical practice, patients who present with metastases but without a known primary cancer are often encountered. It is essential to determine the primary tumor and to treat it accordingly (1). However, occasionally it is difficult to identify the primary tumor site. Therefore, it is only possible to resect or treat the metastases. We report a case of a 43-yearold female patient with a giant origin-unidentified mucinous adenocarcinoma, measuring 143×430x180 mm, penetrating from the pelvic cavity to the subcutaneous tissue. The study was approved by the ethics committee of Wuhan General Hospital of Guangzhou Command, and consent was obtained from the patient involved.

\section{Case report}

A 43-year-old female patient was referred to our hospital with a giant mass on the left buttock (Fig. 1). The mass was of

Correspondence to: Dr Bi-Cheng Zhang, Department of Oncology, Wuhan General Hospital of Guangzhou Command, People's Liberation Army, Wuhan 430070, P.R. China

E-mail: bichengzhang@hotmail.com

Key words: metastatic mucinous adenocarcinoma, unknown origin, pelvis, gastrointestinal tract negligible size when the patient first noticed it (approximately five years ago) and the mass gradually grew to the size of a quail's egg, without evident symptoms, including pain or limited activity. In November 2008, a percutaneous needle biopsy revealed metastatic mucinous adenocarcinoma. Pelvic CT and MRI scans demonstrated two solid-cystic lesions at the bottom of the pelvis, which were further confirmed by pelvic ultrasound to be a complex mass in the left adnexa and a right ovarian cyst. The patient underwent a left ovariectomy and right oophorocystectomy in another hospital, but the mass on the left buttock was not treated. No malignant changes were identified upon histological examination of any of the resected specimens. Following examination, the mass continued to increase in size and limited the patient's quality of life, with symptoms of local discomfort due to pressure stimuli.

In November 2010, a contrast-enhanced CT scan revealed a giant solid-cystic mass with a size of $143 \times 430 \times 180 \mathrm{~mm}$, penetrating from the pelvic cavity to the subcutaneous tissue of the left buttock, in which part of the mass was enhanced by contrast medium (Fig. 2A and B). CT angiography revealed that the mass was fed by the tertiary branches of the left internal iliac artery (Fig. 2C and D). No adjacent bone destruction or lymph node metastasis was identified. Clinical examination revealed a large mass measuring 150x180 mm in the left buttock that was soft, painless, fluctuant, without skin redness, pigmentation or increased temperature.

In mid-November 2010, surgery was performed to remove the mass (Fig. 3A). Intraoperative examination revealed that the mass was solid-cystic, with a capsule adhering to the surrounding tissues. Inside the mass, mucus-like liquid and grape-like tumors were observed. Postoperative pathological examination revealed mucinous adenocarcinoma $(\mathrm{H} \& \mathrm{E}$ staining) (Fig. 3B). Immunohistochemical examination using CDX2 (+++), Ki-67 (++60\%), CA125 (-), TTF1 (-) and Villin $(+++)$ indicated that the mass originated from the epithelial tissues of the digestive tract, but gastrointestinal endoscopes and a postoperative PET-CT scan demonstrated no evidence of cancer of the digestive tract (Fig. 3C and D). Following surgery, the patient received six cycles of adjuvant chemo- 

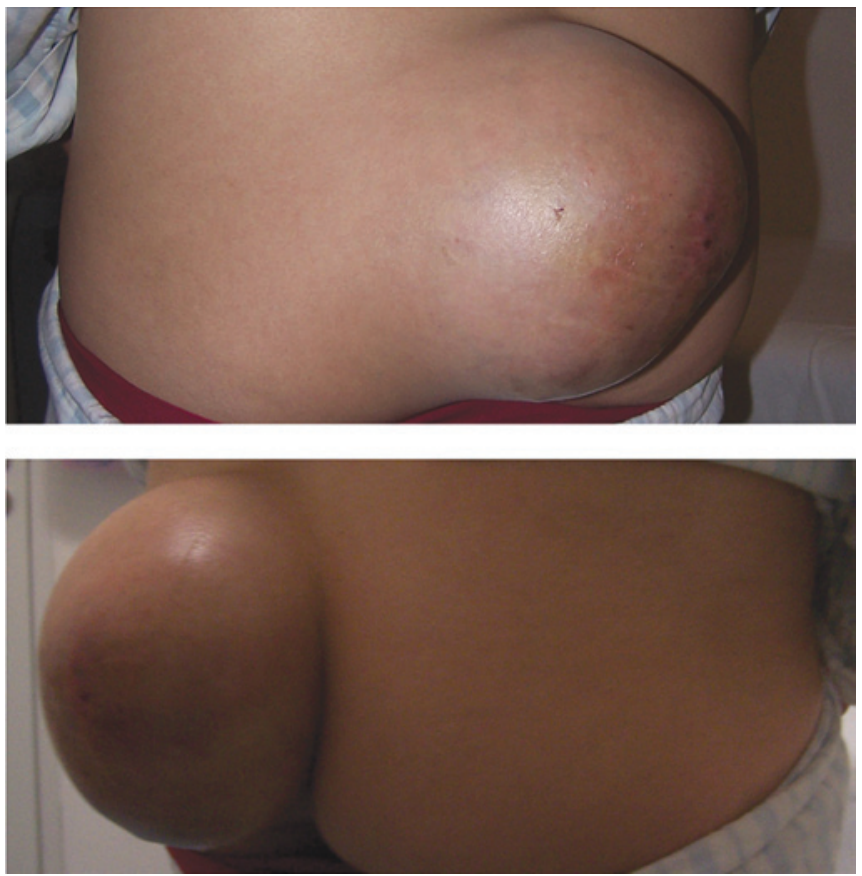

Figure 1. Giant mass on the patient's left buttock.

therapy with oxaliplatin and 5-FU/LV (oxaliplatin $135 \mathrm{mg} /$ $\mathrm{m}^{2}$ day $1 ; 5$-FU $375 \mathrm{mg} / \mathrm{m}^{2} /$ day civ $120 \mathrm{~h} ; \mathrm{LV} 100 \mathrm{mg} / \mathrm{m}^{2}$ day 1-5). Pelvic conformal radiotherapy was performed at a dose of $2.0 \mathrm{~Gy}$ and a total of $60 \mathrm{~Gy}$ over six weeks. However, following surgery, the residual tumor was not reduced as expected, nor did it progress. At present, the patient remains under observation.

\section{Discussion}

In approximately 3 to $5 \%$ of all new cancer cases, it is difficult to determine the primary tumor site, even following extensive imaging and pathology tests $(1,2)$. This difficulty is particularly frequent for large or invasive tumors in the female pelvic region, not only since it is a complex part of the body, but also because it is an area suitable for the acceptance of metastasis (3). In the present case, we were not able to determine the origin of the mass based on imaging information. The origin may be ovarian, uterine, rectum or metastatic. Clinical molecular pathology tests play an increasingly critical role in the differential diagnosis of a tumor of uncertain origin. With regard to metastatic tumors, pathology tests may also provide valuable insights to guide the search for the primary tumor site (4). In the present study, pathological examination of the resected tissue indicated that the mass was a metastatic mucinous adenocarcinoma, which was most likely of gastrointestinal origin. Therefore, we performed gastrointestinal endoscopes, but no malignant change was detected. PET-CT was subsequently performed for whole-body imaging. With the exception of the residual tumor tissue, no other possible tumor was revealed.

Surgical resection was performed on this patient. However, due to the complex anatomy of the pelvis and the difficulty of the surgical approach, the tumor was not completely resected. Therefore, following surgery, the patient was referred for chemotherapy and radiotherapy. In accordance with the
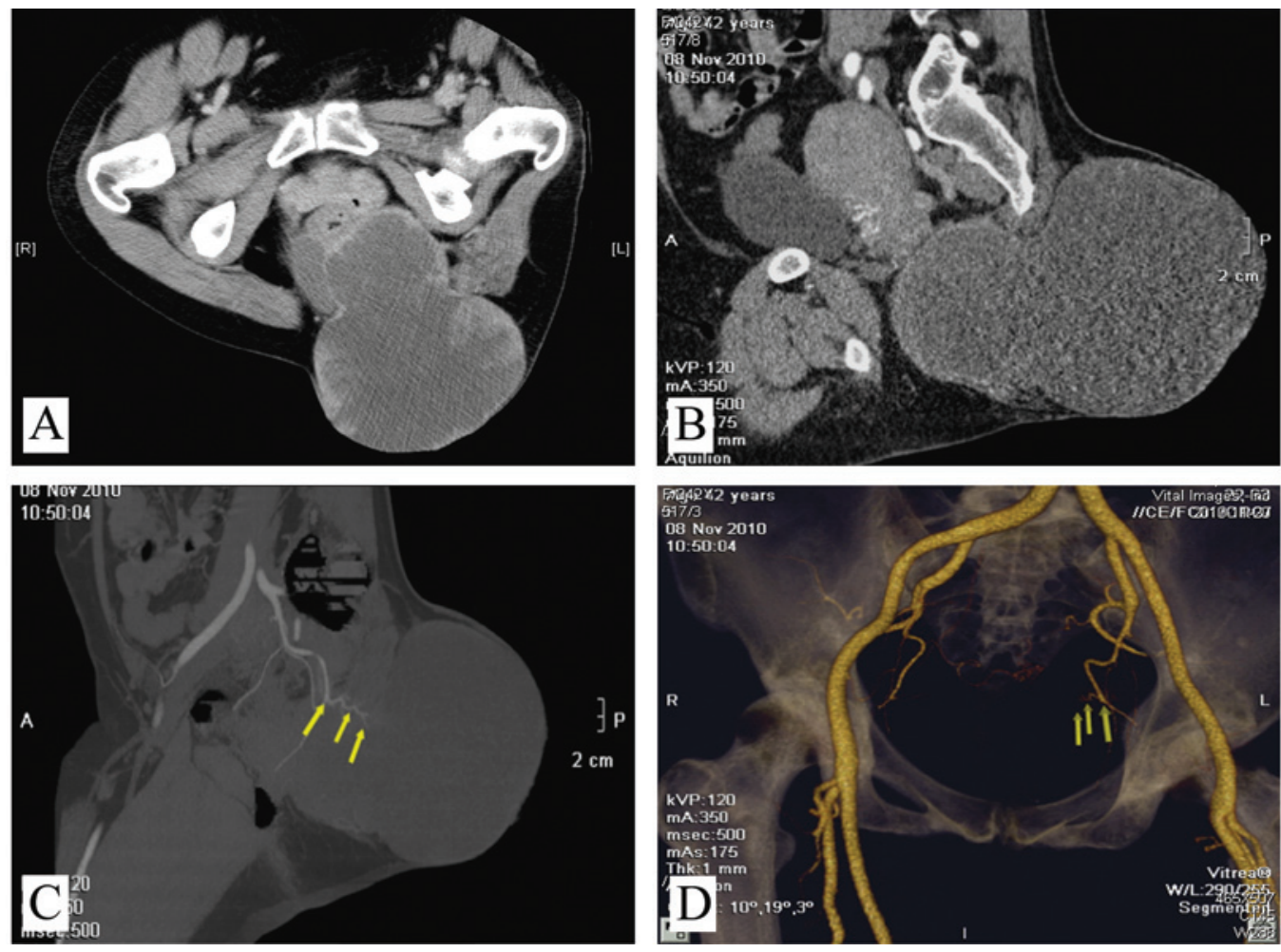

Figure 2. Results of imaging tests. (A) Axial contrast-enhanced CT image. (B) Sagittal contrast-enhanced CT image. (C) Multiplanar reconstruction CT image. (D) Volume-rendered CT angiography image. 

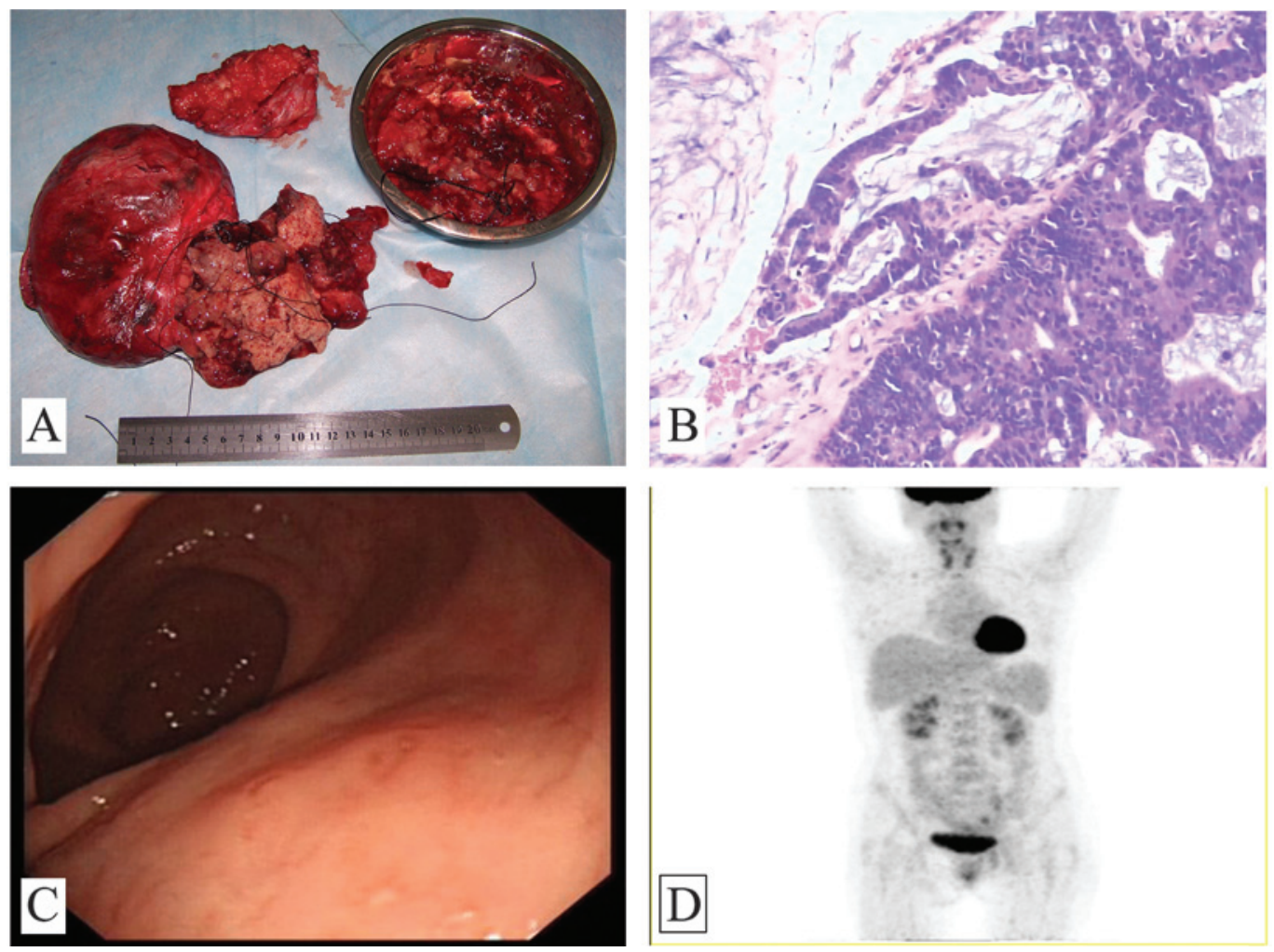

Figure 3. (A) Excised solid-cystic tumor. (B) Pathological examination revealed a mucinous adenocarcinoma (H\&E staining; magnification, x100). (C) Endoscopic image of the rectum. (D) Whole-body imaging using PET-CT.

results of the pathology tests, combination chemotherapy with oxaliplatin and 5-FU/LV (oxaliplatin $135 \mathrm{mg} / \mathrm{m}^{2}$ day $1 ; 5-\mathrm{FU}$ $375 \mathrm{mg} / \mathrm{m}^{2} /$ day civ $120 \mathrm{~h}$; LV $100 \mathrm{mg} / \mathrm{m}^{2}$ day $\left.1-5\right)$, which is commonly used to treat gastrointestinal cancer, was administered (5). During chemotherapy, pelvic conformal radiotherapy was performed using a daily dose of $2.0 \mathrm{~Gy}$ and a total dose of $60 \mathrm{~Gy}$ over six weeks. The response to adjuvant therapy was evaluated with pelvic CT after every two cycles. As previously mentioned, comparison of the pre- and post-treatment CT images indicated no significant changes in the size of the residual tumor. Notably, in terms of growth rate and invasion, the tumor may be diagnosed as low-grade malignant, or even benign. Prior to surgery, the tumor grew continuously at a relatively slow rate, while following surgery and adjuvant therapy the residual tumor almost stopped growing. To date, the mass has existed for over five years and no other metastasis has since been observed. This may be the reason that the residual tumor was not sensitive to postoperative chemotherapy and radiotherapy (6).

In China, due to the limitation of the economic development level and the influence of the coverage of social security, a number of patients are unable to receive immediate and adequate treatment. In terms of the present case, if in 2008 when the patient underwent the first pelvic surgery the mass had been treated properly, it would not have developed to such a huge volume. It is possible that the patient may receive a third surgery in the foreseeable future for the residual tumor following the second surgery at our hospital.

In conclusion, we have reported the case of a giant mucinous adenocarcinoma of unknown origin, which penetrates from the pelvic cavity to the subcutaneous tissue. Since the anatomy surrounding the tumor is complex, surgery was unable to excise the tumor completely. Therefore, further observations and/or treatments are necessary for this patient.

\section{Acknowledgements}

This study was partially supported by the National Foundation of Natural Sciences, China (No. 81101533) and the China Postdoctoral Science Foundation (No. 20100481468).

\section{References}

1. Pavlidis N, Briasoulis E, Hainsworth J and Greco FA: Diagnostic and therapeutic management of cancer of an unknown primary. Eur J Cancer 39: 1990-2005, 2003.

2. Monzon FA, Medeiros F, Lyons-Weiler M and Henner WD: Identification of tissue of origin in carcinoma of unknown primary with a microarray-based gene expression test. Diagn Pathol 5: 3, 2010.

3. Roblick UJ, Bader FG, Lenander C, et al: Undifferentiated pelvic adenocarcinomas: diagnostic potential of protein profiling and multivariate analysis. Int J Colorectal Dis 23: 483-491, 2008.

4. Natoli C, Ramazzotti V, Nappi O, et al: Unknown primary tumors. Biochim Biophys Acta 1816: 13-24, 2011.

5. McRee AJ, Cowherd S, Wang AZ and Goldberg RM: Chemoradiation therapy in the management of gastrointestinal malignancies. Future Oncol 7: 409-426, 2011.

6. Patrone MV, Hubbs JL, Bailey JE and Marks LB: How long have I had my cancer, doctor? Estimating tumor age via Collins' law. Oncology (Williston Park) 25: 38-43, 2011. 